\title{
Confusion in the Garden of Eden
}

(revised version)

\section{Sven Skyum}

DAIMI PB - 2

1973

DEPARTMENT OF COMPUTER SCIENCE UNIVERSITY OF AARHUS

Ny Munkegade, BIdg. 540 DK-8000 Aarhus C, Denmark

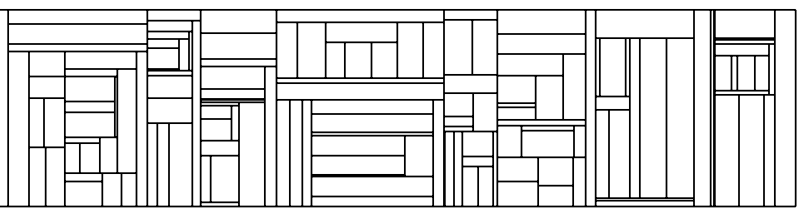


Confusion in the Garden of Eden

by

Sven Skyum

\section{Introduction}

In 1962 Moore [5] showed that the existence of mutually erasable configurations in a two dimensional automata implies the existence of Garden of Eden configurations. In 1963 Myhill [6] showed that the existence of mutually indistinguishable configurations is necessary for the existence of Garden of Eden configurations. Amoroso \& Cooper [1] have shown that Myhill's theorem does not apply to finite configurations but claim, without proof, that both Moore's and Myhill's theorems apply to infinite configurations. Arbib has redefined the concept of Garden of Eden in [2] and has shown that the existence of mutually indistinguishable configurations is both necessary and sufficient for the existence of Garden of Eden configurations. This theorem is not true.

The confusion in the field may be due to different definitions and concepts and prompts the systematic examination of the relation between different characteristics of the global transition function given in this paper.

AMS 1968 subject classifications. Primary 0288, 0280, 9440. KEY words and phrases. Garden of Eden, automata, tesselation automata, cellular automata. 


\section{Definitions and Notations}

A cellular automaton $A$ is a system $\left(1^{n}, 9, \Omega, q_{0}, \sigma\right)$ where:

$1^{\mathrm{n}}$ is the underlying space

$\alpha=\left(a_{1}, a_{2}, \ldots a_{n}\right)$ is a cell.

g: $\mathrm{I}^{\mathrm{n}} \rightarrow\left(\mathrm{I}^{\mathrm{n}}\right)^{\mathrm{m}}$ is the neighbourhood function defined by $g(\alpha)=\left(\alpha+\delta_{1}, \alpha+\delta_{2}, \ldots, \alpha+\delta_{\text {m }}\right)$ where $\delta_{i}(i=1,2, \ldots, m) \in 1^{n}$ is fixed.

$Q$ is a finite set of states.

$q_{0} \in Q$ is a special state called the quiescent state.

$\underline{\sigma}: Q^{m} \rightarrow Q$ is the local transition function.

$\sigma$ is subject to the restriction: $\sigma\left(q_{0}{ }^{11}\right)=q_{0}$

This restriction assures us that no infinite configuration is successor for a finite configuration.

A configuration in $A$ is a mapping $c: 1^{\mathrm{n}} \rightarrow Q$. Let $\underline{b}$ be the set of all configurations in $A$.

A finite configuration in $A$ is a configuration $c$, where the support is finite where $\sup p(c)=\left\{\alpha \in \mathrm{I}^{\mathrm{n}} \mid \mathrm{c}(\alpha) \neq \mathrm{q}_{\mathrm{o}}\right\}$. Let $\underline{b}$ - be the set of all finite configurations in $A$.

The neighbourhood state function $\underline{h}: b \times 1^{\mathrm{n}} \rightarrow Q^{\mathrm{m}}$ is defined by: $\forall c \in b, \forall \alpha \in 1^{n}: h(c, \alpha)=\left(c\left(\alpha+\delta_{1}\right), c\left(\alpha+\delta_{2}\right), \ldots, c\left(\alpha+\delta_{m}\right)\right)$.

The global transition function $\tau: b \rightarrow \zeta$ is defined by: $\forall c \in \zeta, \forall \alpha \in 1^{n}: \tau(c)(\alpha)=\sigma(h(c, \alpha))$.

Let $\tau_{f-}=\tau \mid b_{f}$ be the restriction of $\tau$ to $b_{f} \quad\left(\tau_{f}: b_{f} \rightarrow b_{f}\right)$

$\grave{c}$ is a subconfiguration of $c(\underline{c}<c)$ iff $\left.c\right|_{\operatorname{supp}(\grave{c})}=\left.\grave{c}\right|_{\operatorname{supp}(\grave{c})}$ 
The elements of $G=b_{f} \backslash \tau_{f}\left(b_{f}\right)=b_{f} \backslash \tau\left(b_{f}\right)$ are called Garden of Eden configurations.

This is not equivalent to the original definition of Moore.

$c \in b_{f}$ is strong Garden of Eden iff:

$B S \subset 1^{n}$

1) S finite

2) $\operatorname{supp}(c) \subset s$

3) $\forall \grave{c} \in \ell: c^{*} \in G$ where $c^{*}(\alpha)= \begin{cases}c(\alpha) & \alpha \in S \\ c(\alpha) & \alpha \notin s\end{cases}$

Let $G_{s}$ be the set of all strong Garden of Eden configurations.

$G_{s}=\phi$ iff there are no Garden of Eden configurations of the type defined by Moore.

Arbib defines a configuration $c$ as Garden of Eden when it satisfies: $\forall c \in b_{f}: c<c \Rightarrow c \in G$.

The set $\underline{G}_{a}$, which is the set of configurations satisfying Arbib's definition, is included in $G$.

\section{The global transition function on finite and infinite configurations}

The close connection between properties of configurations and those of the global transition function is shown by the following facts.

(1) $\tau_{f}$ surjective $\Leftrightarrow$ there are no Garden of Eden configurations $(G=\varnothing)$

(2) $\tau_{f}$ injective $\Leftrightarrow$ there are no mutually erasable configurations (see [5]) $\Leftrightarrow$ there are no mutually indistinguishable configurations (see [6]).

A strong GOE configuration is equivalent to a GOE-restriction with respect to $b_{f}$ in the notation of Amoroso, Cooper and Patt. 
In the papers of Moore and Myhill, the proofs depended on the following inequality which was proved for the case $n=2$ and $p=2$. To generalize their proofs to more dimensions and arbitrary finite neighbourhoods, we now give a general proof of the inequality:

\section{느emma:}

$$
\begin{aligned}
& \forall A>1, \forall r>1, \forall n \geq 1, \forall p \geq 1, B k>0: \\
& \left.\left(A^{r}-1\right)^{k}<A^{(k r-p}\right)^{n}
\end{aligned}
$$

Proof:

If $k r>p, 1<i \leq n$ then

$(k r)^{n-i} p^{i}<(k r)^{n-1} \cdot p \wedge\left(\begin{array}{l}n \\ i\end{array}\right) \leq\left(\frac{n}{[n / 2]}\right)$

which means that:

$$
-(k r)^{n-1} \cdot p \cdot\left(\frac{n}{[n / 2]}\right) \cdot n<\sum_{i=1}^{n}(-1)^{i}\left(\begin{array}{l}
n \\
1
\end{array}\right)(k r)^{n-i} \cdot p^{i}
$$

Let $K=r^{n-1} \cdot p \cdot n \cdot\left(\begin{array}{c}n \\ {[n / 2]}\end{array}\right)$

If $k r>p$ we then have

$-K k^{n-1}=-(k r)^{n-1} \cdot p \cdot n\left(\begin{array}{c}n \\ {[n / 2]}\end{array}\right)<\sum_{i=1}^{n}(-1)^{i}\left(\begin{array}{l}n \\ i\end{array}\right)(k r)^{n-i} p^{i}=(k r-p)^{n}-(k r)^{n}$

Choose $k>\max \left(p / r, k / \log _{A}\left(\frac{A^{r^{n}}}{A^{r^{n}}-1}\right)\right)$

$$
\begin{aligned}
& \text { Then we have } \\
& \log _{A}\left(\frac{A^{r}-1}{A^{r}}\right)<-\frac{K}{k} \\
& \Downarrow \\
& A^{n}-1<A^{n}-\frac{K}{K} \\
& \Downarrow\left(A^{r}-1\right)^{k}<A^{(k r)^{n}-K K^{n-1}}<A^{(k r-p)^{n}}
\end{aligned}
$$

\section{Theorem 1:}

$$
G_{S}=\varnothing \Leftrightarrow \tau_{f} \text { injective }
$$


The proof is equivalent ot the proofs found in $[5]$ and $[6]$.

Theorem 2:

$$
b_{f} \subset \tau(b) \Rightarrow \tau_{f} \text { injective }
$$

Proof: Moore's argument from 1962.

Theorem 3:

$$
\tau_{f} \text { injective } \Rightarrow G_{a}=\varnothing
$$

Proof: Myhill's argument from 1963.

\section{Theorem 4:}

$$
6_{f} \backslash \tau(b)=\phi \Leftrightarrow \tau_{f} \text { injective }
$$

\section{Theorem 5:}

$$
\tau \text { injective } \Rightarrow \tau_{f} \text { surjective }
$$

The proofs of theorems 4 and 5 can be found in $[7]$.

Since $\tau_{f}$ surjective $\Rightarrow b_{f} \subset \tau(b)$ and $\tau$ injective $\Rightarrow \tau_{f}$ injective, closuring gives the following diagram:

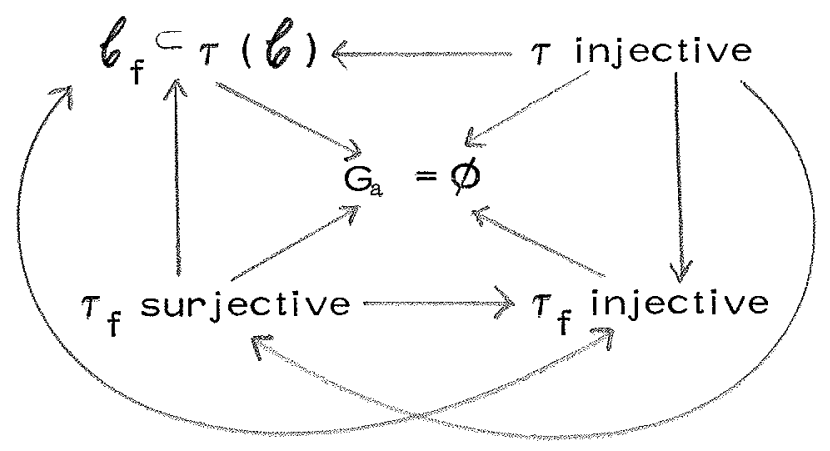


We now give 2 examples which demonstrate that several of the remaining implications are not true.

\section{Example 1:}

$\{1, g(i)=(i, i+1),\{0,1\}, 0,\{00 \rightarrow 0,01 \rightarrow 1,10 \rightarrow 1,11 \rightarrow 0\}\}$

Let $L(c)=\min _{i}\{i \mid c(i)=1\}$ and $R(c)=\max _{i}\{i \mid c(i)=1\}$

$X_{A}($ where $A \subset 1)$ is the characteristic function defined by

$X_{A}(i)=\left\{\begin{array}{lll}1 & \text { if } & i \in A \\ 0 & \text { if } & i \notin A\end{array}\right.$

$\tau$ is not injectivebecause $X_{\phi}$ is the successor of both $X_{1}$ and $i$ tself. On the other hand $I f$ is injective because the following procedure uniquely determines the finite predecessor of $c \in b_{f}$ if it exists.

1) $c(i)=0$ for $i>R(c)$

2) for $i:=R(c)$ downto $L(c)$ :

$\dot{c}(i)=$ if $c(i+1)=1$ then $1-c(i)$ else $c(i)$

3) $d(i)=0$ for $i<L(c)+i$

If we in step 2 replace $L(c)$ with $-\infty$ and drop 3 , we have a procedure for determination of an infinite predecessor. E. g. $\ell_{f} \subset \tau(\zeta)$. However $\tau_{f}$ is not surjective because $X_{\{j\}}=\tau_{f}(c) \Rightarrow L(c)=j+1 \wedge R(c)=j$ which is absurd. Furthermore

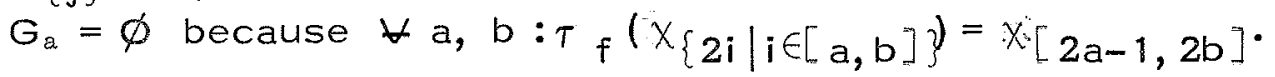

\section{Example 2:}

$\{1, g(i)=(i, i+1),\{0,1\}, 0,\{00 \rightarrow 0,01 \rightarrow 1,10 \rightarrow 1,11 \rightarrow 1\}\}$

$\underline{b}_{f} \Phi \tau(\zeta)$ because $X_{\{j\}}=\tau_{f}(c) \Rightarrow c(j)=1 \Rightarrow x_{\{j\}}(j-1)=1$ which is absurd.

$G_{a}=\phi$ because $a \leq b: \tau_{f}(x[a, b])=X_{[a-1, b]}$.

Finally $\tau_{f}$ is not injective because $\tau_{f}\left(X_{\{1,3\}}\right)=\tau_{f}\left(X_{\{1,2,3\}}\right)=$ $X\{0,1,2,3\}$. 
We have the following diagram:

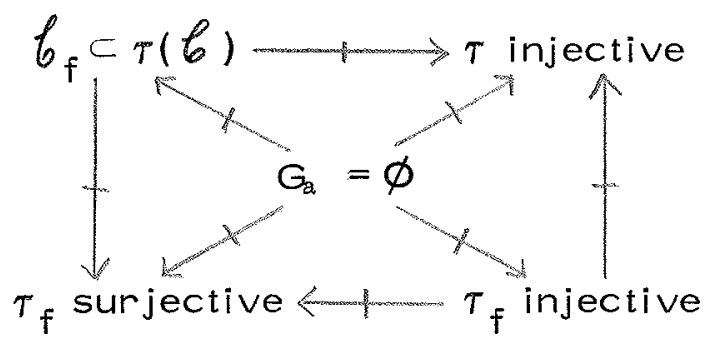

From this it appears among other things that the definition of Garden of Eden appearing in this paper (and in many others) is too weak for the Garden of Eden theorem to have a converse, and Arbib's definition is too strong for the theorem to be true.

\section{Open Problem:}

Is it true or not that $\tau_{f}$ surjective $\Rightarrow \tau$ injective ? 


\section{BIBLIOGRAPHY}

[1] S. Amoroso \& G. Cooper:

The Garden of Eden Theorem for Finite Configurations. (Proc. of the Amer. Math. Soc. 26, 1970, pp. 158-164)

[2] M. A. Arbib:

Theories of Abstract Automata.

(Prentice Hall 1969)

[3] W. Burks:

Essays on Cellular Automata.

(University of Illinois Press 1970)

[4] E.F. Codd:

Cellular Automata.

(ACM Monograph Series, Academic Press 1968)

[5] E.F. Moore:

Machine models of Self-Reproduction.

(Proc. of Symp. in Apll. Math., vol. 14, 1962)

[6] J. Myhill:

The Converse of Moore's Garden of Eden Theorem.

(Proc. of the Amer. Math. Soc., vol. 14, 1963, pp. 685-686)

[7] D. Richardson:

Tessellations with Local Transformations.

(Journal of Comp. and System Sci. 6, 1972 , pp. 373-388) 